\title{
ON FINITE LOOPS WHOSE INNER MAPPING GROUPS ARE ABELIAN
}

\author{
MARKKU NiEMENMAA
}

\begin{abstract}
Loops are nonassociative algebras which can be investigated by using their multiplication groups and inner mapping groups. If the inner mapping group of a loop is finite and Abelian, then the multiplication group is a solvable group. It is clear that not all finite Abelian groups can occur as inner mapping groups of loops. In this paper we show that certain finite Abelian groups with a special structure are not isomorphic to inner mapping groups of finite loops. We use our results and show how to construct solvable groups which are not isomorphic to multiplication groups of loops.
\end{abstract}

\section{INTRODUCTION}

If $Q$ is a groupoid, then we say that $Q$ is a loop if $Q$ has a neutral element $e$ and each of the two equations $a x=b$ and $y a=b$ has a unique solution for any $a, b \in Q$. The mappings $L_{a}(x)=a x$ (left translation) and $R_{a}(x)=x a$ (right translation) are permutations on $Q$ for every $a \in Q$ and the permutation group $M(Q)=\left\langle L_{a}, R_{a}\right.$ : $a \in Q\rangle$ is called the multiplication group of $Q$. It is obvious that $M(Q)$ is transitive in $Q$ and the stabilisers of elements of $Q$ are conjugated in $M(Q)$. The stabiliser of the neutral element $e$ is denoted by $I(Q)$ and this subgroup of $M(Q)$ is called the inner mapping group of $Q$. These two notions linking loops to groups were defined by Bruck $[2]$ and he was the first to investigate the structure of loops by using group theory.

In this paper, which is a continuation of $[\mathbf{6}, \mathbf{7}, \mathbf{9}, \mathbf{1 0}]$, we are interested in the structure of inner mapping groups of loops. In [11] we managed to prove that if $I(Q)$ is a finite Abelian group, then $M(Q)$ is solvable. Now it is clear that some finite Abelian groups can occur as inner mapping groups of loops while other finite Abelian groups are never isomorphic to inner mapping groups of loops. We thus started to investigate the following problem: Which finite Abelian groups are (are not) isomorphic to inner mapping groups of loops? It is not very difficult to see that $I(Q)=1$ if and only if $Q$ is an Abelian group. We also know [7, 10] that $I(Q)$ is cyclic if and only if $Q$ is an Abelian group. In [9] we showed that for a finite loop $Q, I(Q)$ can not be isomorphic to $C_{n} \times D$, where $C_{n}$ is a cyclic group of order $n$ and $D$ is a finite Abelian group

Received 31st October, 2001

Copyright Clearance Centre, Inc. Serial-fee code: 0004-9727/02 \$A2.00+0.00. 
such that $n$ and $|D|$ are relatively prime. Later on, Kepka [6] managed to show that this result is true for infinite loops, too. The purpose of this paper is to show that the following two results hold:

(1) If $Q$ is a finite loop, then $I(Q)$ is never isomorphic to the direct product $C_{p^{k}} \times C_{p}$, where $p$ is an odd prime number and $k \geqslant 2$.

(2) Let $Q$ be a finite loop and $p \neq q$ be two prime numbers such that $p$ is odd and $q$ does not divide $|Q|$. Then $I(Q)$ is not isomorphic to $\left(C_{p^{k}} \times C_{p}\right) \times$ $D$, where $k \geqslant 2$ and $D$ is an Abelian $q$-group.

Here we wish to point out that if $Q$ is a group, then $I(Q)$ is the group of inner automorphisms of $Q$. The question which finite Abelian groups occur as inner automorphism groups of groups was completely solved by Baer [1].

Many properties of loops can be reduced to the properties of connected transversals in the multiplication group. The notion of connected transversals was introduced by Kepka and Niemenmaa [10] in 1990 and since then it has been at the heart of much of the current research on loops and their relation to groups. This paper is no exception: we apply the theory of connected transversals when proving our main results. Section 2 contains basic information about connected transversals, gives the characterisation theorem of multiplication groups of loops and contains other preliminary results which are needed later. In Section 3 we prove our main results, first in purely group theoretical terms (using connected transversals), and after that we give the loop theoretical interpretation of our results in Section 4. Finally, by using this information, we show how to construct examples of solvable groups which are not isomorphic to multiplication groups of loops.

Our notation is standard. For a general overview about the relation between loops and groups we refer to $[2,10,13,14,15]$. The reader interested in the history of loop theory should have a look at the article by Pflugfelder [12] and those who wish to know about the applications of nonassociative algebras are advised to read the articles [3, $4,8]$.

\section{GROUPS, LOOPS AND CONNECTED TRANSVERSALS}

Let $Q$ be a loop and consider the groups $M(Q)$ and $I(Q)$ and the left and right translations defined in the introduction. If we write $A=\left\{L_{a}: a \in Q\right\}$ and $B=\left\{R_{a}: a \in Q\right\}$, then the commutator subgroup $[A, B] \leqslant I(Q)$ and $A$ and $B$ are left transversals to $I(Q)$ in $M(Q)$. If $1<K \leqslant I(Q)$, then $K$ is not a normal subgroup of $M(Q)$. Finally, $M(Q)=\langle A, B\rangle$.

We then consider the corresponding situation in groups in general. Let $H$ be a subgroup of $G$ and let $A$ and $B$ be two left transversals to $H$ in $G$. We say that $A$ and $B$ are $H$-connected if $[A, B] \leqslant H$. In fact, $H$-connected transversals are both left 
and right transversals ([10, Lemmas 2.1 and 2.2]). By $H_{G}$ we denote the core of $H$ in $G$, that is, the largest normal subgroup of $G$ contained in $H$. If $H_{G}=1$, we say that $H$ is core-free in $G$. The relation between multiplication groups of loops and connected transversals is given by

THEOREM 2.1. A group $G$ is isomorphic to the multiplication group of a loop if and only if there exist a subgroup $H$ satisfying $H_{G}=1$ and $H$-connected transversals $A$ and $B$ such that $G=\langle A, B\rangle$.

For the proof, see [10, Theorem 4.1].

In the following lemmas we assume that $A$ and $B$ are $H$-connected transversals in $G$.

Lemma 2.2. If $H_{G}=1$, then $N_{G}(H)=H \times Z(G)$.

Lemma 2.3. If $C \subseteq A \cup B$ and $K=\langle H, C\rangle$, then $C \subseteq K_{G}$.

LEMma 2.4. If $H_{G}=1$, then $Z(G) \subseteq A \cap B$.

For the proofs, see [10, Lemma 2.5 and Proposition 2.7] and [7, Lemma 1.4]. The reader should observe that from Lemma 2.3 it immediately follows that $K=K_{G} H$. In the following four lemmas we assume that $G=\langle A, B\rangle$. As usual, $p$ denotes a prime number.

Lemma 2.5. If $H$ is a cyclic subgroup of $G$, then $G^{\prime} \leqslant H$.

LEMmA 2.6. If $H \cong C_{p} \times C_{p}$, then $G^{\prime} \leqslant N_{G}(H)$.

LEMmA 2.7. If $G$ is a finite group and $H \cong C_{n} \times D$, where $n>1, D$ is Abelian and $\operatorname{gcd}(n,|D|)=1$, then $H_{G}>1$.

Lemma 2.8. If $G$ is a finite group and $H$ is Abelian, then $H$ is subnormal in $G$.

For the proofs, see [7, Theorem 2.2], [11, Lemma 4.2], [9, Theorem 2.3], and [9, Lemma 2.1].

If $G$ is a finite group, then the Frattini subgroup $\Phi(G)$ is the intersection of all maximal subgroups of $G$. Clearly, $\Phi(G)$ is a characteristic subgroup of $G$. We need

Lemma 2.9. If $G$ is a $p$-group, then $\Phi(G)=G^{\prime}\left\langle x^{p}: x \in G\right\rangle$.

For the proof, see [5, pp. 272-273].

LEMma 2.10. If $[a, b]$ commutes with $a$ and $b$, then $(a b)^{n}=a^{n} b^{n}[b, a]^{\left(\begin{array}{l}n \\ 2\end{array}\right)}$.

For the proof, see [5, pp. $253-254]$.

\section{MaIN ThEOREMS}

Throughout this section we assume that $G$ is a finite group, $H$ is an Abelian 
subgroup of $G$ with a special structure and there exist $H$-connected transversals $A$ and $B$ such that $G=\langle A, B\rangle$.

THEOREM 3.1. Let $H \cong C_{p^{k}} \times C_{p}$ where $p$ is an odd prime number and $k \geqslant 2$. Then $H_{G}$ is not trivial.

Proof: Let $G$ be a counterexample of smallest possible order. Thus $H_{G}=1$ and from Lemmas 2.2 and 2.8 it follows that $N_{G}(H)=H \times Z(G)$ and $Z(G)>1$. If $z \in Z(G)$ and $|z|=r$ ( $r$ is a prime number), then by considering $G /\langle z\rangle$ and $H\langle z\rangle /\langle z\rangle$, we may assume that there exists a normal subgroup $K$ of $G$ such that $\langle z\rangle\langle K \leqslant H\langle z\rangle$ (here $K$ is the largest normal subgroup of $G$ contained in $H\langle z\rangle$ ). If $r \neq p$, then $K$ has a Sylow $p$-subgroup which is normal in $G$, hence $H_{G}>1$. Thus we may assume $r=p$ and we may conclude that $Z(G)$ is a $p$-group.

If $K$ contains an element $x \in H$ of order $p^{l}(l \geqslant 2)$, then by Lemma $2.9, \Phi(K)$ is a nontrivial subgroup of $H$ and $\Phi(K)$ is normal in $G$, which is not possible. Thus $K$ is an elementary Abelian $p$-group. If $k \geqslant 3$, then we consider $G / K$ and $H K / K$. If $H K / K$ is cyclic, then it follows from Lemma 2.5 that $H K=H\langle z\rangle$ is normal in $G$, hence $\Phi(H\langle z\rangle)$ is normal in $G$. Since this is not possible, we use induction and conclude that there exists a normal subgroup $L$ of $G$ such that $K<L \leqslant H K=H\langle z\rangle$. As this is not possible, we may assume that $k=2$.

We write $H=\langle y\rangle \times\langle x\rangle$, where $|y|=p^{\overline{2}}$ and $|x|=p$. Thus $K=\left\langle y^{p}\right\rangle \times\langle z\rangle$ and $H K / K$ is elementary Abelian of order $p^{2}$. From Lemma 2.6 we conclude that $G^{\prime} \leqslant N_{G}(H K)$. In what follows, we denote the group $N_{G}(H K)$ by $E$. Clearly, $E$ is normal in $G$. As the core of $H K / K$ in $G / K$ is trivial, it follows from Lemma 2.2 that $N_{G / K}(H K / K)=H K / K \times Z(G / K)$. We write $M / K=Z(G / K)$ and then $E=H K M$, where $H K \cap M=K$ and $M$ is a normal subgroup of $G$. By Lemma 2.4, $Z(G / K) \subseteq A K / K \cap B K / K$ and thus $M \subseteq A K \cap B K$.

Now $E=C H=D H$, where $C \subseteq A$ and $D \subseteq B$. Furthermore, it is easy to see that $M \subseteq C K=D K$ and, by Lemma 2.4, we must have $M=C\left\langle y^{p}\right\rangle=D\left\langle y^{p}\right\rangle$. As $H K$ is normal in $E$, it follows that $\Phi(H K)=\left\langle y^{p}\right\rangle$ is normal in $E$ (and in $M$ ). Since $[C, D] \leqslant H \cap M=\left\langle y^{p}\right\rangle$, we have $M^{\prime} \leqslant\left\langle y^{p}\right\rangle$. Since $M^{\prime}$ is normal in $G$, we conclude that $M^{\prime}=1$, hence $M$ is an Abelian group. Thus $K=\left\langle y^{p}, z\right\rangle \leqslant Z(E)$.

Since $E^{\prime} \leqslant H K$ and $E^{\prime}$ is normal in $G$, we conclude that $E^{\prime} \leqslant K$. We write $W=\left\langle g \in E: g^{p}=1\right\rangle$. It is immediate that $W$ contains $x, y^{p}$ and $z$ and $W$ is a normal subgroup of $G$. If $g, h \in W$, then $(g h)^{p}=g^{p} h^{p}[h, g]^{\left(\begin{array}{l}p \\ 2\end{array}\right)}=1$ by Lemma 2.10 (remember that $p$ is odd) and thus $W=\left\{g \in E: g^{p}=1\right\}$. If we consider the group $G / W$ and its cyclic subgroup $H W / W$, then it follows from Lemma 2.5 that $G^{\prime} \leqslant H W$, which means that $H W$ is normal in $G$. If $h \in H$ and $w \in W$, then we use Lemma 2.10 and get $(h w)^{p}=h^{p} w^{p}[w, h]^{\left(\begin{array}{l}p \\ 2\end{array}\right)}=h^{p} \in\left\langle y^{p}\right\rangle$. Thus $(H W)^{p}=\left\langle t^{p}: t \in H W\right\rangle=\left\langle y^{p}\right\rangle$ and since $(H W)^{p}$ is a characteristic subgroup of $H W$, we conclude that $\left\langle y^{p}\right\rangle$ is normal 
in $G$, a contradiction. The proof is complete.

The following two lemmas will be needed in the proof of Theorem 3.4.

Lemma 3.2. Let $p \neq q$ be two prime numbers. If $H \cong C_{p} \times D$, where $D$ is an Abelian Sylow $q$-subgroup of $G$, then $G^{\prime} \leqslant H$.

Proof: Let $G$ be a minimal counterexample. By Lemma 2.7, $H_{G}>1$. If $\left|H_{G}\right|$ divides $|D|$, then we are done by induction (or by Lemma 2.5). If $\left|H_{G}\right|=p t$, where $1<t \leqslant|D|$, then we have a nontrivial subgroup $K$ of $D$ which is normal in $G$ and again we are ready. We still have to consider the case $\left|H_{G}\right|=p$. Let $F=N_{G}(D)$ and let $M$ be a maximal subgroup of $G$ such that $M \geqslant F$. Clearly, $F \geqslant H$. If $H=M$, then from Lemma 2.8 it follows that $H$ is normal in $G$, hence $G^{\prime} \leqslant H$. If $H<M$, then we use Lemma 2.3 and get $M_{G}>1$. Thus $H M_{G} / M_{G}=M / M_{G}$ is subnormal in $G / M_{G}$ by Lemma 2.8 , which means that $M$ is normal in $G$. By the Frattini argument, $G=M N_{G}(D)=M$, a contradiction. Thus $G=F$ and it follows that $D$ is normal in $G$. But then $H=H_{G} D$ is normal in $G$ and $G^{\prime} \leqslant H$.

Lemma 3.3. Let $p \neq q$ be two prime numbers. If $H \cong\left(C_{p} \times C_{p}\right) \times D$, where $D$ is an Abelian Sylow $q$-subgroup of $G$, then $G^{\prime} \leqslant N_{G}(H)$.

Proof: Our proof is by induction on $|G|$ and we first assume that $H_{G}>1$. By using Lemmas 2.5, 2.6 and 3.2 and the fact that $G$ is a minimal counterexample, we may conclude that $G^{\prime} \leqslant N_{G}(H)$ with one exception: the case $H_{G} \cong C_{p} \times C_{p}$ has to be investigated separately. We write $F=N_{G}(D)$ and as in the proof of the previous lemma, we conclude that $G^{\prime} \leqslant H_{G} D=H$.

We then assume that $H_{G}=1$. From Lemmas 2.2 and 2.8 it follows that $N_{G}(H)$ $=H \times Z(G)$ and $Z(G)>1$. Let $z \in Z(G)$ such that $|z|=r$, where $r$ is a prime number. By induction, we have $G^{\prime} \leqslant N_{G}(H\langle z\rangle)=L$. If $r \neq q$, we consider the group $E=L N_{G}(D)$. As $E$ is normal in $G$, we apply the Frattini argument and thus $G=E N_{G}(D)=L N_{G}(D)=E$. Clearly, $D$ is normal in $L$, hence $D$ is normal in $G$. As $H_{G}=1$, this is not possible. Thus we must have $r=q$ which means that $z$ is a $q$-element. As $D$ is a Sylow $q$-subgroup of $G$, it is clear that $z \in D$. But then $H_{G}>1$, a contradiction. The proof is complete.

Now we are ready to prove

THEOREM 3.4. Let $p \neq q$ be two prime numbers. If $H \cong\left(C_{p^{k}} \times C_{p}\right) \times D$, where $p$ is an odd prime, $k \geqslant 2$ and $D$ is an Abelian Sylow $q$-subgroup of $G$, then $H_{G}$ is not trivial.

Proof: Again we assume that $G$ is a minimal counterexample, hence $H_{G}=1$. We proceed as in the beginning of the proof of Theorem 3.1 and we conclude that there is an element $z \in Z(G)$ such that $|z|=r$, where $r$ is a prime number. We also have 
a normal subgroup $K$ of $G$ such that $K>\langle z\rangle$ is the largest normal subgroup of $G$ contained in $H\langle z\rangle$.

Assume first that $r \neq p$. If $p$ divides $|K|$, then $K$ has a nontrivial normal Sylow $p$-subgroup $P \leqslant H$ and it is clear that $P$ is normal in $G$. If $p$ does not divide $|K|$, then the Sylow $p$ - subgroup of $H K / K$ is isomorphic to $C_{p^{k}} \times C_{p}$ and by Theorem 3.1 or by induction, we conclude that there exists a normal subgroup $N$ of $G$ such that $K<N \leqslant H K=H\langle z\rangle$, a contradiction.

Thus we may assume that $r=p$. If $K$ has a Sylow $q$-subgroup $Q$, then $Q \leqslant H$ is normal in $G$ and therefore $q$ does not divide $|K|$. If $K$ contains an element $x \in H$ of order $p^{l}(l \geqslant 2)$, then $\Phi(K)$ is a nontrivial subgroup of $H$ which is normal in $G$. As this is not possible, we conclude that $K$ is an elementary Abelian $p$-group.

If the Sylow $p$-subgroup of $H K / K$ is cyclic or isomorphic to $C_{p l} \times C_{p}(l \geqslant 2)$, then by Lemma 2.7 or by induction there exists a normal subgroup $M$ of $G$ such that $K<M \leqslant H K$. This is not possible and therefore $H K / K \cong\left(C_{p} \times C_{p}\right) \times$ $D$. We conclude that $k=2$ and $H=(\langle y\rangle \times\langle x\rangle) \times D$, where $|y|=p^{2}$ and $|x|=$ $p$. Furthermore, $K=\left\langle y^{p}, z\right\rangle$. By Lemma 3.3, $(G / K)^{\prime} \leqslant N_{G / K}(H K / K)$, which means that $G^{\prime} \leqslant N_{G}(H K)$. Then consider the group $T=N_{G}(H K) N_{G}(D)$. As $T \geqslant G^{\prime}$, it follows that $T$ is normal in $G$. We use the Frattini argument and see that $G=N_{G}(H K) N_{G}(D)$. Since $D$ is normal in $N_{G}(H K)$, it follows that $D$ is normal in $G$. But then $H_{G}>1$, a contradiction. The proof is complete.

\section{LOOP THEORETICAL RESULTS}

In Theorems 3.1 and 3.4 we have two purely group theoretical results which tell us how the structure of an Abelian subgroup with connected transversals determines the subgroup not to be core-free. When we combine Theorem 3.1 with Theorem 2.1 we immediately have the following result in loop theory.

COROLlary 4.1. Let $p$ be an odd prime number and $Q$ a finite loop. Then the inner mapping group $I(Q)$ can not be isomorphic to $C_{p^{k}} \times C_{p}$, where $k \geqslant 2$.

Theorem 3.4 also has an interpretation in loop theory.

Corollary 4.2. Let $Q$ be a finite loop and $p, q$ two different prime numbers such that $p$ is odd and $q$ does not divide $|Q|$. Then the inner mapping group $I(Q)$ can not be isomorphic to $\left(C_{p^{k}} \times C_{p}\right) \times D$, where $k \geqslant 2$ and $D$ is an Abelian $q$-group.

Proof: Suppose the claim is false and we have a finite loop $Q$ whose inner mapping group $I(Q)$ is isomorphic to the Abelian group given in the corollary. Now $M(Q)=A I(Q)=B I(Q)$ and $|A|=|B|=|Q|$ (here $A$ and $B$ denote the sets of left and right translations introduced in the beginning of Section 2). As $q$ does not divide $|Q|$, it follows that $D$ is a Sylow $q$-subgroup of $M(Q)$. By Theorem 3.4, $I(Q)$ 
is not core-free in $M(Q)$ contradicting the properties of $I(Q)$ (see the beginning of Section 2). The proof is complete.

If $G$ is a finite nonabelian group and $H$ is a nontrivial proper subgroup of $G$, then we consider the following conditions on $H:$ 1) $H_{G}>1$, 2) $\left.N_{G}(H)>H Z(G), 3\right) H$ is cyclic, 4) $H \cong C_{p^{k}} \times C_{p}$, where $p$ is odd and $k \geqslant 2$. From the properties of the inner mapping group of a loop (see Theorems 2.1 and 4.1 and lemmas 2.2 and 2.5) it follows that if each nontrivial proper subgroup of $G$ satisfies at least one of the conditions 1 ), $2), 3$ ) and 4), then $G$ is not isomorphic to the multiplication group of a loop. We now use these conditions in the following example.

EXAMPLE. Let $R$ be a cyclic group of order $r, T$ a cyclic group of order $t$ and let $\operatorname{gcd}(t, r)=1$. Assume further that $p$ is an odd prime, $p^{k}(k \geqslant 2)$ divides $\mid$ Aut $(R) \mid, p$ divides $\mid$ Aut $(T) \mid$ and $p$ divides neither $r$ nor $t$. Now let $U$ be the semidirect product of $R$ and the cyclic group of order $p^{k}$ and let $V$ be the semidirect product of $T$ and the cyclic group of order $p$. Then $G=U \times V$ is a finite group of order $r t p^{k+1}$ and $Z(G)=1$. By looking at the subgroups of this group one sees that each nontrivial proper subgroup satisfies at least one of the four conditions listed before. Thus $G$ is not isomorphic to the multiplication group of a loop.

FinAL REMARKS. In the light of the results given in our corollaries, it is quite natural to ask whether these results hold when $p=2$. It would also be interesting to prove Corollary 4.2 in the following more general form: $I(Q)$ is not isomorphic to $\left(C_{p^{k}} \times C_{p}\right) \times D$, where $k \geqslant 2$ and $D$ is a finite Abelian group such that $p$ does not divide $|D|$.

\section{REFERENCES}

[1] R. Baer, 'Erweiterung von Gruppen und ihren Isomorphismen', Math. Z. 38 (1934), $375-416$.

[2] R.H. Bruck, 'Contributions to the theory of loops', Trans. Amer. Math. Soc. 60 (1946), 245-354.

[3] J. Denes and A. Keedwell, 'A new authentication scheme based on latin squares', Discrete Math. 106/107 (1992), 157-161.

[4] T. Foguel and A. Ungar, 'Gyrogroups and the decomposition of groups into twisted subgroups and subgroups', Pacific J. Math. 197 (2001), 1-11.

[5] B. Huppert, Endliche Gruppen I, Die Grundlehren der Mathematischen Wissenschaften 134 (Springer-Verlag, Berlin, Heidelberg, New York, 1967).

[6] T. Kepka, 'On the abelian inner permutation groups of loops', Comm. Algebra. 26 (1998), 857-861.

[7] T. Kepka and M. Niemenmaa, 'On loops with cyclic inner mapping groups', Arch. Math. 60 (1993), 233-236.

[8] C. Moore, D. Therien, F. Lemieux, J. Berman and A. Drisko, 'Circuits and expressions with nonassociative gates', J. Comput. System Sci. 60 (2000), 368-394. 
[9] M. Niemenmaa, 'On the structure of the inner mapping groups of loops', Comm. Algebra 24 (1996), 135-142.

[10] M. Niemenmaa and T. Kepka, 'On multiplication groups of loops', J. Algebra 135 (1990), 112-122.

[11] M. Niemenmaa and T. Kepka, 'On connected transversals to abelian subgroups', Bull. Austral. Math. Soc. 49 (1994), 121-128.

[12] H.O. Pflugfelder, 'Historical notes on loop theory', Comment. Math. Univ. Carolin. 41 (2000), 359-370.

[13] J.D. Phillips, 'A note on simple groups and simple loops', in Proceedings of the Groups (Korea 1998) (Walter de Gruyter, Berlin, New York, 2000), pp. 305-317.

[14] J.D.H. Smith, 'Loops and quasigroups: aspects of current work and prospects for the future', Comment. Math. Univ. Carolin. 41 (2000), 415-427.

[15] A. Vesanen, 'Solvable loops and groups', J. Algebra 180 (1996), 862-876.

Department of Mathematical Sciences

University of Oulu, PL 3000

90014 Oulu

Finland 